\title{
Efficient generation of ultra-intense few-cycle radially polarized laser pulses
}

\author{
Sergio Carbajo, ${ }^{1,2,3, *}$ Eduardo Granados, ${ }^{1}$ Damian Schimpf, ${ }^{2}$ Alexander Sell, ${ }^{1}$ \\ Kyung-Han Hong, ${ }^{1}$ Jeffrey Moses, ${ }^{1}$ and Franz X. Kärtner ${ }^{1,2,3}$ \\ ${ }^{1}$ Research Laboratory of Electronics, and Department of Electrical Engineering and Computer Science, \\ Massachusetts Institute of Technology, 77 Massachusetts Avenue, Cambridge, Massachusetts 02139, USA \\ ${ }^{2}$ Center for Free-Electron Laser Science, Deutsches Elektronen Synchrotron, and Department of Physics, \\ University of Hamburg, Notkestraße 85, 22607 Hamburg, Germany \\ ${ }^{3}$ Hamburg Center for Ultrafast Imaging, Luruper Chaussee 149, 22607 Hamburg, Germany \\ *Corresponding author: scarbajo@mit.edu
}

Received February 4, 2014; revised March 16, 2014; accepted March 17, 2014; posted March 17, 2014 (Doc. ID 205882); published April 14, 2014

\begin{abstract}
We report on efficient generation of millijoule-level, kilohertz-repetition-rate few-cycle laser pulses with radial polarization by combining a gas-filled hollow-waveguide compression technique with a suitable polarization mode converter. Peak power levels $>85 \mathrm{GW}$ are routinely achieved, capable of reaching relativistic intensities $>10^{19} \mathrm{~W} / \mathrm{cm}^{2}$ with carrier-envelope-phase control, by employing readily accessible ultrafast high-energy laser technology. (c) 2014 Optical Society of America

OCIS codes: (190.7110) Ultrafast nonlinear optics; (320.5520) Pulse compression; (060.5060) Phase modulation; (140.3300) Laser beam shaping; (260.5430) Polarization.

http://dx.doi.org/10.1364/OL.39.002487
\end{abstract}

The generation of radially polarized laser pulses with relativistic intensities can unfold new horizons in the nonlinear optical regime where laser-matter interactions are dominated by the relativistic motion of the electrons. High-energy, few-cycle radially polarized sources remain relatively unexplored today. Traditionally, the generation of pulses with relativistic intensities has been limited to chirp pulse amplification-based techniques with linearly polarized lasers [1-ㅜㅇ. Radially polarized beams present several advantages compared to those with linear or circular polarization. They can be focused significantly tighter, hence entailing less demanding peak powers to reach any given optical fluence, and exhibit up to two times stronger longitudinal field intensities than transversely at the focal region $[\underline{4}, \underline{5}]$. These features are relevant not only to a wide variety of applications requiring relativistic intensities but also to those uniquely sensitive to electromagnetic vector symmetry about the propagation axis or to those exhibiting a polarization vortex at the center of the beam, such as atomic and molecular dynamics []], high-resolution second-harmonic generation [7], enhanced solid-state laser amplification [8], micromachining [9] , and particle acceleration [10-12].

In this Letter, we focus on experimentally demonstrating a highly efficient linear-to-radial polarization mode conversion induced at the output of a few-cycle millijoule-level hollow-waveguide compressor where nearly transform-limited few-cycle pulses with radial polarization are achieved. This scheme results in a radially polarized $\sim 3$-cycle laser centered at $800 \mathrm{~nm}$ routinely operating at peak and average power levels of $85 \mathrm{GW}$ and $2 \mathrm{~W}$, respectively, comprising the shortest pulse duration and the highest peak- and average-power radially polarized source reported to date [13-15]. Moreover, exploiting the intensity scalability in the few-cycle regime offers the additional benefit of reaching out to physical processes dependent on the carrier phase relative to the pulse envelope.
The drive laser (Coherent Legend Elite Duo USP) generates linearly polarized, up to $5 \mathrm{~mJ}$ pulses at $3 \mathrm{kHz}$ repetition rate with $35 \mathrm{~nm}$ bandwidth centered at $800 \mathrm{~nm}$ wavelength [Fig. 1]. The carrier-envelope phase of the amplifier output can be locked within $200 \mathrm{mrad}$ rms (10 shot integration). The pulses are coupled into an Ar-filled dielectric hollow waveguide, where they undergo spectral broadening due to the combined effect of self-phase modulation and group-delay dispersion, a topic extensively studied in the literature [16]. The hollow waveguide has an inner diameter of $500 \mu \mathrm{m}$, an outer diameter of $2 \mathrm{~mm}$, and a length of $1 \mathrm{~m}$. The Ar gas flows along the waveguide in a pressure gradient geometry. The optical output end of the waveguide is set with constant laminar flow of Ar while the input is kept at 0.15 Torr, inducing an end-to-end net pressure drop of $10 \mathrm{psi}$. The combination of a large waveguide core radius relative to the optical wavelength with a reduced opticalinput gas pressure enables high pulse energy before detrimental self-focusing and ionization occurs at the input of the fiber, thereby maximizing spectral broadening and throughput.

For the purposes of this study, we spectrally broaden $1.5 \mathrm{~mJ}$ input pulses from the Ti:sapphire amplifier to $130 \mathrm{~nm}$ full width at half-maximum (FWHM) bandwidth [Fig. 2a]. The efficiency of the spectral broadening process is $60 \%$, measured from input to output of the hollow waveguide, in close proximity to the maximum achievable value of $68 \%$. The major throughput loss contributors are identified as waveguide attenuation, mode-coupling efficiency, and $Q$ factor, or the energy in the fundamental hybrid $\mathrm{EH}_{11}$ mode relative to the total energy [17]. Spectral broadening is a nonlinear process strongly dependent on the input pulse intensity and shape, the laser pointing, and gas pressure. Therefore, a high spectral broadening efficiency in a large coreradius waveguide is indicative of maximized self-phase modulation; increased spatiotemporal output beam 
a

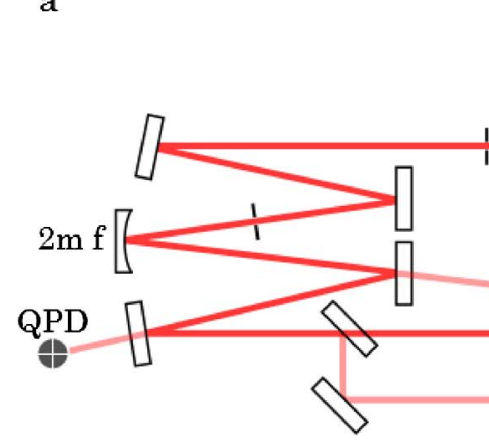

b

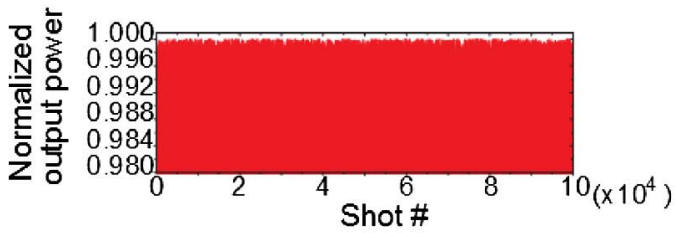

$\lambda=800 \mathrm{~nm}$
$\Delta \tau \sim 38 \mathrm{fs}$

Ti:Sapph

$\mathrm{E}=1.5 \mathrm{~mJ}$

@ $3 \mathrm{kHz}$

Fig. 1. a, schematic layout of the pressure gradient hollow-waveguide compressor setup and the polarization modeconverter. Ar gas is laminarly flown in the counterpropagating direction of the laser beam. b, short-term output intensity fluctuations over more than $3 \mathrm{~s}$ accounting for $<0.5 \%$ over the average intensity value due to active pointing stabilization of the input beam. For optimum alignment, the fluctuations are comparable to the those inherent to the Ti:sapphire amplifier system.

quality, intensity, and spectral stability; and minimized cross-phase modulation between the fundamental and other copropagating higher order leaky waveguide modes. The output of the hollow waveguide exhibits excellent long- and short-term stability for practical applications. The short-term fluctuations of the broadened pulses account for $<0.5 \% \mathrm{rms}$ of the output intensity, shown in Fig. 1b. With active stabilization of the input laser pointing the system operates stably for several hours. The broadened fundamental mode is assumed to exit the waveguide nearly diffraction limited and exhibits a quasi-Gaussian profile, 5 mrad divergence, and linear polarization. The pulses are temporally compressed, employing a suitable set of double-chirp mirrors that compensate cumulative second- and third-order dispersions of 270 and $190 \mathrm{fs}^{3}$, respectively. The throughput of the compressor is $86 \%$, and the FWHM pulse duration measured at the output is $8 \mathrm{fs}$, corresponding to a value 1.1 times the transform-limited pulse duration.

The approach of hollow-waveguide broadening after conversion from linear to radial polarization is disadvantageous for several reasons. Efficient coupling and propagation of the radially polarized $\left(\mathrm{TM}_{01}\right)$ ultrafast laser pulses in hollow waveguides has only been demonstrated at low energies of the order of a few microjoules [18]. While cylindrically symmetric vector modes $\mathrm{TE}_{0 q}$ and $\mathrm{TM}_{0 q}$ exist in circular waveguides for the entire wavelength spectrum, the dominant nonsymmetric transverse modes in the infrared region are the hybrid $\mathrm{EH}_{p q}$ modes. For spectral broadening of high-energy pulses above a few tens of microjoules the required waveguide core radius becomes significantly larger. This condition imposes an underperformance of the $\mathrm{TM}_{01}$ mode relative to the fundamental $\mathrm{EH}_{11}$ mode that arises primarily from a lower coupling efficiency, approximately 14\% lower than the $\mathrm{EH}_{11}$ mode assuming a Gaussian beam, as shown in Fig. 3, and higher propagation losses in a hollow dielectric waveguide. The latter can be set to be arbitrarily small by utilizing a sufficiently large core radius since the attenuation constant is directly proportional to $\lambda^{2} / a^{3}$, where $\lambda$ is the wavelength and $a$ is the waveguide radius [19]. However, the ratio of attenuation constants between any given TM mode and hybrid mode is given by $\alpha_{\mathrm{TM}_{0 k}} / \alpha_{\mathrm{EH}_{n m}}=2\left(\nu^{2}\left(u_{0 k} / u_{n m}\right)^{2} /\left(\nu^{2}+1\right)\right)$, where $\nu$ is the medium-normalized index of refraction and $U_{i j}$ is the $i j$ th root of $J_{1}\left(U_{i j}\right)=0$, where $J_{1}$ is the Bessel function of the first kind. The first unfavorable implication of these conditions is that the minimum possible propagation loss of the $\mathrm{TM}_{01}$ mode will generally be restricted to be $>3 \times$ larger than that of the fundamental $\mathrm{EH}_{11}$ mode, and thus the maximum achievable
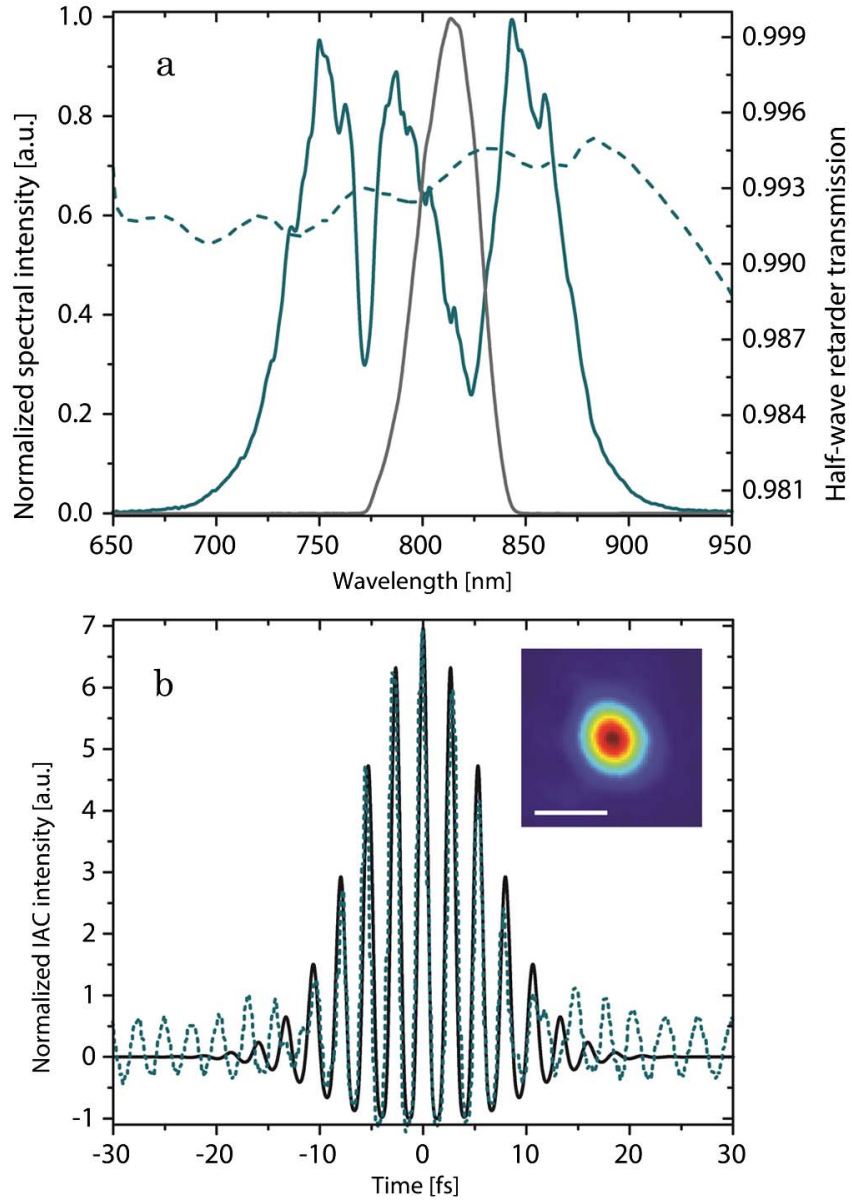

Fig. 2. a, normalized input spectrum (gray), normalized output spectrum (solid cyan), and $\lambda / 2$ waveplate transmission curves (dashed cyan). b, measured interferometric autocorrelator (IAC) signal (dashed cyan) and fitted IAC signal with a $8.06 \pm$ 0.15 fs FWHM pulse duration and waveguide output far-field beam profile (inset); bar is $5 \mathrm{~mm}$. 


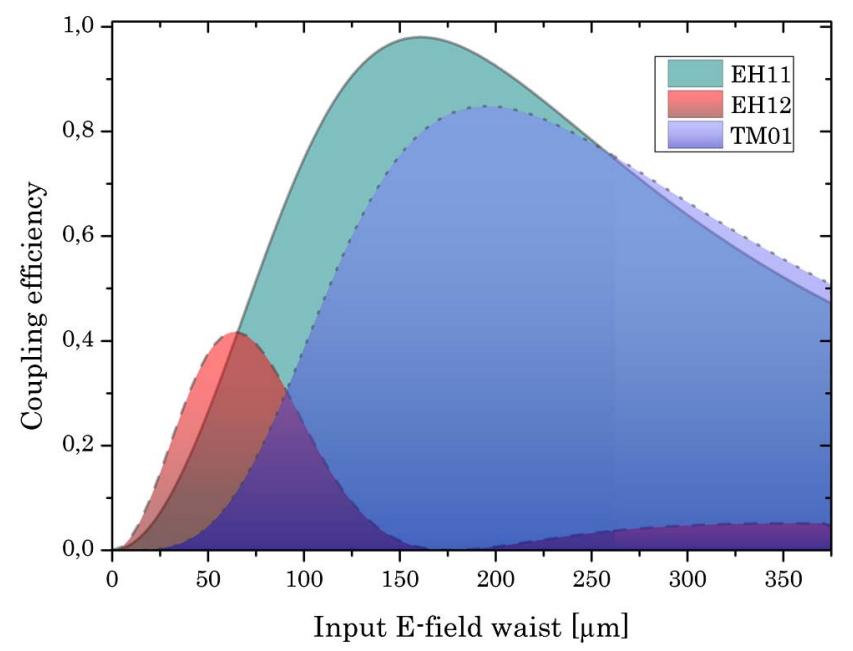

Fig. 3. Computed coupling efficiency as a function of electric field beam waist of linearly polarized Gaussian $\mathrm{EH}_{11}$ (solid) and $\mathrm{EH}_{12}$ (dashed) beams and linearly polarized Gaussian beam externally converted to $\mathrm{TM}_{01}$ (dotted) mode.

broadening factor and efficiency will be reduced. Another possible consequence is that energy launched into the preferred $\mathrm{TM}_{01}$ mode will transfer toward all other modes, especially toward the more dominant ones, generally leading to additional losses and manifesting an increased undesirable cross talk between comparably strong propagating modes.

Practically, these limitations can be overcome by introducing a broadband polarization-state converter at the hollow-waveguide output in order to preserve the high throughput and broadening factor of the gas-filled hollow waveguide corresponding to the $\mathrm{EH}_{11}$ mode operation. We demonstrate this concept by employing a custom segmented achromatic waveplate consisting of eight $\lambda / 2 \pm$ $2 \%$ retarders over a $650-950 \mathrm{~nm}$ bandwidth range, each arranged with the slow birefringence axis rotated at $\pm 11.25^{\circ}, \pm 37.75^{\circ}, \pm 56.25^{\circ}$, and $\pm 78.75^{\circ}$ angles and the same thickness [20]. The mode converter has a clear aperture of $20 \mathrm{~mm}$ in diameter and exhibits a measured throughput $>96 \%$ for the spectrally broadened pulses. As illustrated in Fig. 2, the converted radially polarized pulses from the segmented waveplate are compressed again down to 8 fs by slightly modifying the compressor to account for the increased dispersion, which comprises approximately total second- and third-order dispersions of $320 \mathrm{fs}^{2}$ and $210 \mathrm{fs}^{3}$, respectively. The retarder-induced phase difference across the bandwidth of these pulses remains small relative to their wavenumber, thus making it possible to compress them to a nearly transformlimited duration. However, this effect will become prominent when approaching the single-cycle limit. Our result is directly transferable to the case of azimuthal polarization by introducing a quarter-wave retarder. Because of its cylindrical symmetry, the degree of polarization $(\mathcal{P})$ is evaluated over the transverse $0^{\circ}, \pm 45^{\circ}$, and $90^{\circ}$ axes, where we find excellent polarization purity (Fig. 4). The Stokes parameters $S_{0}, S_{1}, S_{2}$, and $S_{3}$ are calculated directly from recorded images at transverse $0^{\circ}, \pm 45^{\circ}$, and $90^{\circ}$ analyzer orientations. Using these calculated parameters, the polarization purity is verified quantitatively to exceed $93 \%$, where $\mathcal{P}=S_{0}^{-1} \sqrt{S_{1}^{2}+S_{2}^{2}+S_{3}^{2}}[\underline{21}$.
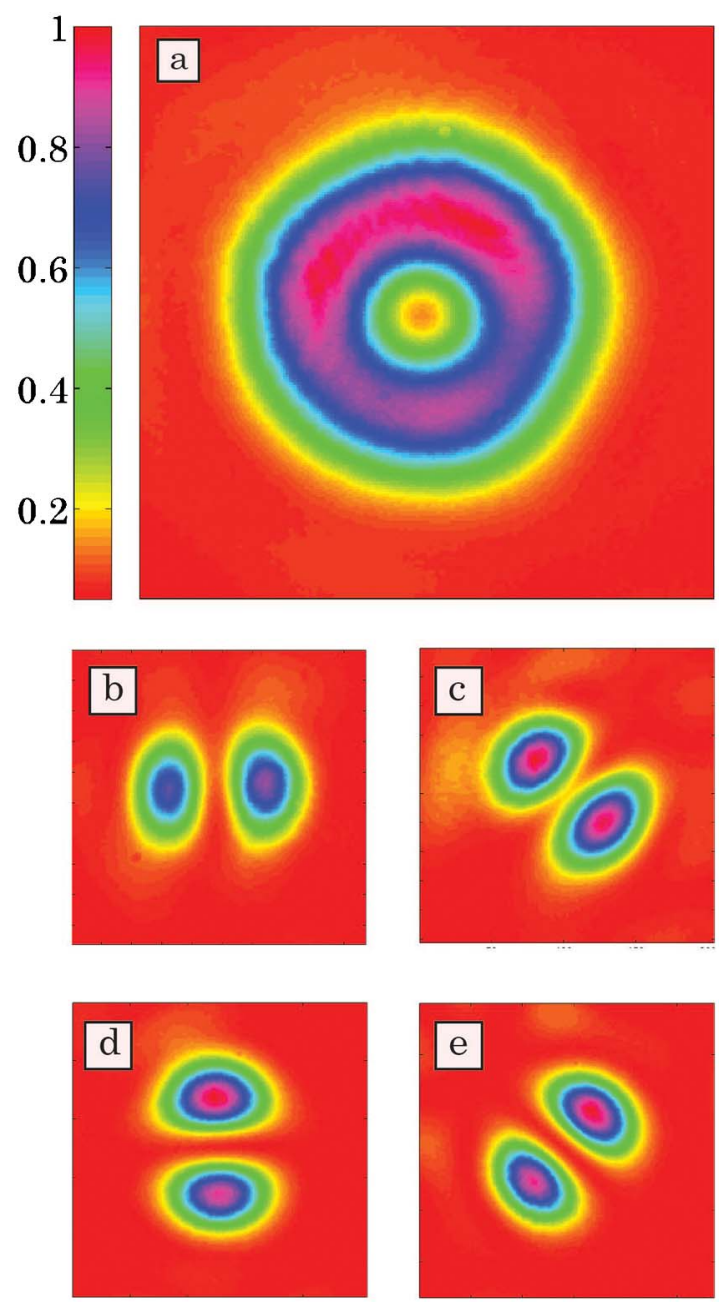

Fig. 4. Normalized transverse far-field intensity profile of $8 \mathrm{fs}$ radially polarized beam for a, full beam; $b, 0^{\circ}$-angle; $c, 45^{\circ}$-angle; d, $90^{\circ}$-angle; and e, $135^{\circ}$-angle components.

In summary, we have experimentally demonstrated a simple and robust technique to efficiently produce high peak- and average-power few-cycle radially polarized beams by adequately combining well-established optical technologies. We find that a segmented achromatic waveplate employed for postspectral broadening is a feasible method for polarization mode conversion of energetic few-cycle pulses. The excellent spatial quality and high radial-polarization purity of the beam make it now possible for these pulses to reach fluences well into the relativistic intensity regime up to $\sim 6 \cdot 10^{19} \mathrm{~W} / \mathrm{cm}^{2}$. Further progress in the compression efficiency of the laser system will soon extend the outcome of this work to more energetic and shorter radially polarized pulses and scale up reachable intensities by an order of magnitude. This demonstration has now become an enabling technology for emerging applications such as vacuum electron acceleration to relativistic energies.

This work has been supported by the DARPA AXIS Program under contract N66001-1-11-4192, the U.S. Air Force Office of Scientific Research through contracts FA9550-12-1-0080 and FA9550-12-1-0499, and by the excellence cluster "The Hamburg Centre for Ultrafast Imaging-Structure, Dynamics and Control of Matter 
at the Atomic Scale" of the Deutsche Forschungsgemeinschaft. A. Sell acknowledges support by a Feodor-Lynen fellowship of the Alexander von Humboldt Foundation and E. Granados by an IKERBASQUE fellowship from Spain.

\section{References}

1. M. D. Perry and G. Mourou, Science 264, 917 (1994).

2. K. H. Hong, B. Hou, J. A. Nees, E. Power, and G. A. Mourou, Appl. Phys. B 81, 447 (2005).

3. O. Albert, H. Wang, D. Liu, Z. Chang, and G. Mourou, Opt. Lett. 25, 1125 (2000).

4. R. Dorn, S. Quabis, and G. Leuchs, Phys. Rev. Lett. 91, 233901 (2003).

5. K. Youngworth and T. Brown, Opt. Express 7, 77 (2000).

6. L. Novotny, M. R. Beversluis, K. S. Youngworth, and T. G. Brown, Phys. Rev. Lett. 86, 5251 (2001).

7. D. P. Biss and T. G. Brown, Opt. Lett. 28, 923 (2003).

8. I. Moshe, S. Jackel, and A. Meir, Opt. Lett. 28, 807 (2003).

9. C. Hnatovsky, V. G. Shvedov, N. Shostka, A. V. Rode, and W. Krolikowski, Opt. Lett. 37, 226 (2012).

10. L. J. Wong and F. X. Kärtner, Opt. Express 18, 25035 (2010).

11. L. Cicchitelli, H. Hora, and R. Postle, Phys. Rev. A 41, 3727 (1990).
12. J. Rosenzweig, A. Murokh, and C. Pellegrini, Phys. Rev. Lett. 74, 2467 (1995).

13. W. D. Kimura, G. H. Kim, R. D. Romea, L. C. Steinhauer, I. V. Pogorelsky, K. P. Kusche, R. C. Fernow, X. Wang, and Y. Liu, Phys. Rev. Lett. 74, 546 (1995).

14. S. Payeur, S. Fourmaux, B. E. Schmidt, J. P. MacLean, C. Tchervenkov, F. Lagare, M. Piche, and J. C. Kieffer, Appl. Phys. Lett. 101, 041105 (2012).

15. M. Bock, J. Brunne, A. Treffer, S. König, U. Wallrabe, and R. Grunwald, Opt. Lett. 38, 3642 (2013).

16. M. Nisoli, S. De Silvestri, and O. Svelto, Appl. Phys. Lett. 68, 2793 (1996).

17. E. Granados, L.-J. Chen, C.-J. Lai, K.-H. Hong, and F. X. Kärtner, Opt. Express 20, 9099 (2012).

18. A. A. Ishaaya, C. J. Hensley, B. Shim, S. Schrauth, K. W. Koch, and A. L. Gaeta, Opt. Express 17, 18630 (2009).

19. E. A. J. Marcatili and R. A. Schmeltzer, Bell Syst. Tech. J. 43 , 1783 (1964).

20. G. Machavariani, Y. Lumer, I. Mosche, A. Meir, and S. Jackel, Opt. Lett. 32, 1468 (2007).

21. M. Born and E. Wolf, Principles of Optics (Cambridge University, 1999). 